\title{
Role of Biochemical Investigation in Prediction of Biliary Etiology in Acute Pancreatitis
}

\author{
Tanka Prasad Bohara,' Anuj Parajuli,' Mukund Raj Joshi' \\ 'Department of Surgery, Kathmandu Medical College Teaching Hospital, Sinamangal, Kathmandu, Nepal.
}

Introduction: Acute pancreatitis is common clinical presentation. Gall stone disease and alcohol consumption are most common etiologies. Detection of biliary etiology is important in order to provide definite management in form of cholecystectomy to prevent further attacks. Sensitivity for abdominal ultrasound to detect cholelithiasis is decreased to $67-87 \%$ in presence of acute pancreatitis. Difference in biochemical investigations of acute biliary and non-biliary pancreatitis has been proposed to increase the suspicion of biliary etiology.

Methods: Sixty patients admitted with diagnosis of acute pancreatitis from March 2012 to December 2012 were included in the study. The relation between etiology with age, sex, admission serum amylase and liver function test were evaluated.

Results: Out of 60 patients $38(63.33 \%)$ and $22(36.66 \%)$ had biliary and non-biliary etiology respectively. Biliary pancreatitis was more common in females ( 25 vs. 3). Distribution of severity was comparable between both groups. Biliary pancreatitis group had significantly higher amylase level (3466.42 vs. 1987.5, p 0.003) whereas values of liver function test were higher in biliary pancreatitis though not statistically significant.

Conclusions: A simple, rapid and accurate prediction of biliary etiology of acute pancreatitis can be provided by changes in biochemical parameters.

Keywords: Acute Pancreatitis; Biliary Pancreatitis; Serum Amylase.

\section{INTRODUCTION}

Acute Pancreatitis (AP) is an acute inflammatory process of pancreas that frequently affects the peripancreatic tissue and less frequently affects the systemic organs. ${ }^{1,2}$ Identification of biliary cause of pancreatitis is important to provide definite management in form of cholecystectomy to prevent further attacks.

Abdominal ultrasound (AUS) is the most important tool to detect cholelithiasis with sensitivity of almost $100 \%$ in uncomplicated situation which decreases to $67-87 \%$ in AP. ${ }^{3-5}$ Endoscopic Ultrasound (EUS) has higher rate of

Correspondence:Dr Tanka Prasad Bohara, Department of Surgery, Kathmandu Medical College Teaching Hospital, Sinamangal, Kathmandu, Nepal. Email: tankaprasad.bohara@gmail.com, Phone: 9841352378. 
detection of biliary etiology resulting in $75 \%$ of initially diagnosed idiopathic AP to be biliary pancreatitis. ${ }^{5-7}$ But EUS is not yet available in Nepal. Several biochemical investigations such as bilirubin, Alkaline Phosphatase (ALP), Alanine Transaminase (ALT), Aspartate transaminase (AST), serum lipase and amylase have been proposed to identify a biliary etiology. ${ }^{8-10}$

Aim of the study was to investigate the difference in biochemical parameters between biliary and non-biliary acute pancreatitis and its role in detection of biliary etiology.

\section{METHODS}

A cross sectional study was conducted in Department of Surgery, Kathmandu Medical College Teaching Hospital with diagnosis of first episode of acute pancreatitis from March 2012 to December 2012 were included in the study. Patients with chronic liver disease and those not giving consent were excluded. Written informed consent was taken from the patient. Ethical approval was taken from institutional review board.

Diagnosis of acute pancreatitis was made if two of the following three features were present (1) abdominal pain consistent with AP; (2) serum amylase (or lipase) more than three times normal value; (3) characteristic findings of AP on AUS, Contrast Enhanced Computed Topography (CECT) abdomen or Magnetic Resonance Imaging (MRI).

Diagnosis of biliary and alcoholic pancreatitis was confirmed by presence of gall stones on AUS and history of alcohol binge without gall stones respectively. Patient with dyslipidemia, hypercalcemia, history of drug intake known to cause acute pancreatitis, trauma and Endoscopic Retrograde Cholangiopancratography (ERCP) in the absence of other cause were considered to have AP pancreatitis related to these causes. Patient without any identifiable cause were labelled as idiopathic pancreatitis. Severity of pancreatitis was classified as per Revised Atlanta Classification, ${ }^{11}$ as mild acute pancreatitis, moderately severe pancreatitis and severe acute pancreatitis. Demographic data, history, relevant physical findings, serum amylase, Liver Function Test (LFT), AUS, CECT Abdomen and MRI (if done) findings were noted. Patients were divided into two groups based upon etiology as biliary and non-biliary group. Chi square test and Mann - Whitney test were used two compare parametric and non-parametric variables between two groups. SPSS 15.0 was used to analyse the data. $P$ value $<0.05$ was considered significant.

\section{RESULTS}

Sixty patient were admitted with diagnosis of acute pancreatitis (AP) out of which $38(63.33 \%)$ and 22 $(36.66 \%)$ had biliary and non-biliary etiology. The etiology in non-biliary groups included alcoholic, traumatic, malignancy and idiopathic (Table 1).

\begin{tabular}{|lc|}
\hline Table 1. Etiologies in non- biliary group. \\
\hline Etiology & Number (Percentage) \\
Alcohol & $9(40.9 \%)$ \\
Idiopathic & $11(50 \%)$ \\
Traumatic & $1(4.54 \%)$ \\
Malignancy & $1(4.54 \%)$ \\
\hline
\end{tabular}

As expected number of female patients was higher and statistically significant in biliary group (25 vs. 3, $P=0.00$ ) (Figure 1).

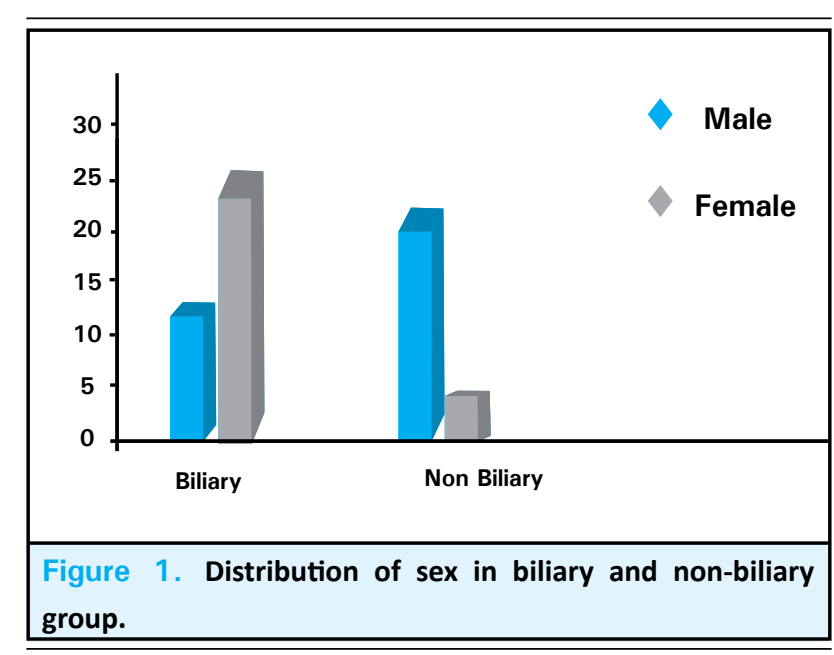


Distribution of severity of pancreatitis was comparable in both groups (Figure 2).

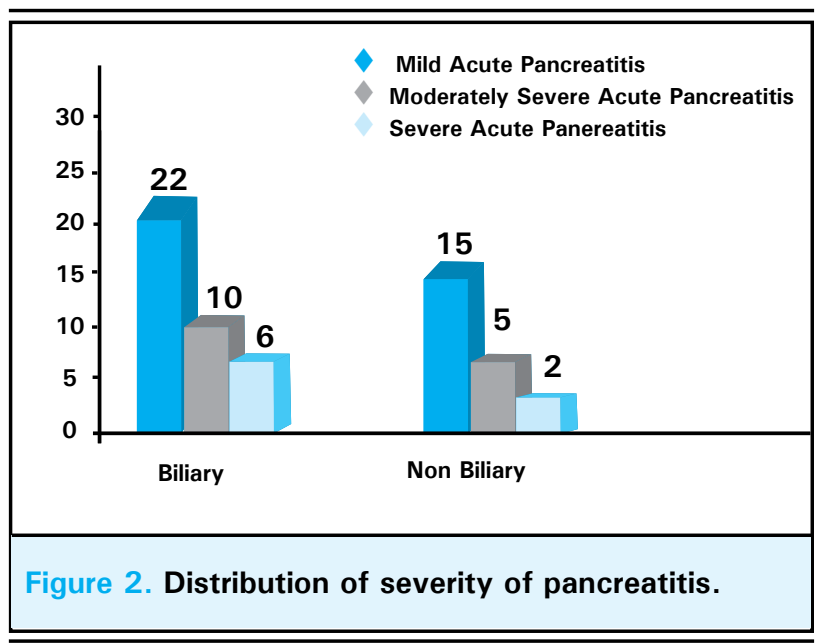

Means of total bilirubin, direct bilirubin, AST, ALT and ALP were higher in biliary group but could not reach statistically significant level. However mean of serum amylase was significantly higher in biliary group (Table 2).

\begin{tabular}{|llll|}
\hline \multicolumn{4}{l}{ Table 2. Comparisons of means of various biochemical } \\
parameters. \\
Investigation & Biliary & Non Biliary & P value \\
Total Bilirubin & $2.15 \mathrm{mg} / \mathrm{dl}$ & $1.65 \mathrm{mg} / \mathrm{dl}$ & .064 \\
Direct Bilirubin & $1.08 \mathrm{mg} / \mathrm{dl}$ & $0.86 \mathrm{mg} / \mathrm{dl}$ & .024 \\
AST & $200.47 \mathrm{U} / \mathrm{I}$ & $115.18 \mathrm{U} / \mathrm{I}$ & .043 \\
ALT & $173.23 \mathrm{U} / \mathrm{l}$ & $98.5 \mathrm{U} / \mathrm{I}$ & .054 \\
ALP & $433.13 \mathrm{U} / \mathrm{l}$ & $345.27 \mathrm{U} / \mathrm{I}$ & .151 \\
Serum Amylase & $3466.42 \mathrm{U} / \mathrm{l}$ & $1987.5 \mathrm{U} / \mathrm{I}$ & .003 \\
\hline
\end{tabular}

\section{DISCUSSION}

Most common etiologies of AP are gallstone disease and alcohol consumption which accounts for almost 80 to $90 \%$ of the cases. Other etiologies include medicines, infections, metabolic disorders, trauma, ERCP etc. ${ }^{2}$ Distinction of biliary pancreatitis is important in order to provide specific treatment like cholecystectomy or ERCP and sphinterotomy in unfit patient. The association between rise in hepatic transaminase level and biliary pancreatits was first suggested by McMohan and Pickford. ${ }^{12}$ ALT is the most commonly used to predict biliary etiology and is thought to be the most useful of the available biochemical investigations in predicting gall stone etiology of AP and variable level of ALT has been identified with Positive Predictable value (PPV) between 98 to $100 \%{ }^{8,13}$ However in current study ALT levels was higher in biliary group but not statistically significant ( $128 \mathrm{U} / \mathrm{L}$ vs $545 \mathrm{U} / \mathrm{L}, \mathrm{P}=0.54$ ) and PPV and Negative Predictable Value (NPV) was $47 \%$ and $81 \%$ respectively when cut off value of $150 \mathrm{U} / \mathrm{L}$ was considered.

Ammori, ${ }^{9}$ group used AUS, EUS and post mortem finding to diagnose cholelithiasis and reported that LFT was $99.9 \%$ sensitive and $100 \%$ specific with PPV of $100 \%$ and NPV of $85.7 \%$. Gungor et al, ${ }^{14}$ used Magnetic Resonance Cholangiopancreaticography (MRCP), ERCP and Intraoperative cholangiogram to differentiate biliary and non-biliary pancreatitis and concluded that total bilirubin, direct bilirubin, amylase and lipase levels may be used in prediction of biliary pancreatitis. Anderson et al $^{13}$ suggested that ALT rather than AUS alone is an important investigation to justify cholecystectomy in patient presenting with AP.

Biliary etiology of AP should be considered in female patients with deranged LFT and highly raised serum amylase level even if initial AUS do not detect cholelithiasis. Negative AUS for cholelithiasis in this subset of patient should not be accepted. Follow up AUS is recommended and if still negative EUS can be considered but it is time consuming, may not be easily available and there is always risk of further attack while waiting for the investigation. Definite management of cholelithiasis may be based upon early derangement of biochemical investigations. EUS is still recommended in patients with idiopathic pancreatitis with normal LFT.

Current study has some limitations. Diagnosis of biliary cause was mostly made AUS and CECT and MRCP (if done). There were 11 patients of idiopathic pancreatitis, if they were subjected to further investigations like EUS, biliary cause could have been identified. This may be reason why difference in LFT could not reach significant level. Different time of presentation from the onset of AP might have also altered the level of biochemical investigation.

\section{CONCLUSIONS}

A simple, rapid and accurate prediction of cholelithiasis as etiology of AP can be provided by changes in biochemical parameters. EUS is not always necessary to detect biliary cause of AP.

\section{ACKNOWLEDGEMENTS}

We would like to thank the ethical committee of Kathmandu Medical College Teaching Hospital for their guidance. 


\section{REFERENCES}

1. Trikudanathan G, Navneethan U, Vege SS. Current controversies in fluid resuscitation in acute pancreatitis: a systematic review. Pancreas. 2012 Aug;41(6):827-34.

2. Banks PA. Epidemiology, natural history, and predictors of disease outcome in acute and chronic pancreatitis. Gastrointest Endosc. 2002 Dec;56(6 Suppl):S226-30.

3. Hessler PC, Hill DS, Deforie FM, Rocc FM. High accuracy sonographic recognition of gallstones. AJR Am J Roentgenol. 1981 Mar;136(3):517-20.

4. Alexakis N, Lombard M, Raraty M, Ghaneh P, Smart HL, Gilmore I, et al. When is pancreatitis considered to be of biliary origin and what are the implications for management? Pancreatology. 2007;7(2-3):131-41.

5. Liu CL, Lo CM, Chan JK, Poon RT, Fan ST. EUS for detection of occult cholelithiasis in patients with idiopathic pancreatitis. Gastrointest Endosc. 2000 Jan;51(1):28-32.

6. Frossard JL, Sosa-Valencia L, Amouyal G, Marty O, Hadengue A, Amouyal P. Usefulness of endoscopic ultrasonography in patients with "idiopathic" acute pancreatitis. Am J Med. 2000 Aug 15;109(3):196-200.

7. Norton SA, Alderson D. Endoscopic ultrasonography in the evaluation of idiopathic acute pancreatitis. Br J Surg. 2000 Dec;87(12):1650-5.
8. Ammori BJ, Boreham B, Lewis P, Roberts SA. The biochemical detection of biliary etiology of acute pancreatitis on admission: a revisit in the modern era of biliary imaging. Pancreas. 2003 Mar;26(2):e32-5.

9. Tenner S, Dubner H, Steinberg W. Predicting gallstone pancreatitis with laboratory parameters: a meta-analysis. Am J Gastroenterol. 1994 Oct;89(10):1863-6.

10. Davidson BR, Neoptolemos JP, Leese T, Carr-Locke DL. Biochemical prediction of gallstones in acute pancreatitis: a prospective study of three systems. Br J Surg. 1988 Mar;75(3):213-5.

11. Banks PA, Bollen TL, Dervenis C, Gooszen HG, Johnson CD, Sarr MG, et al. Classification of acute pancreatitis--2012: revision of the Atlanta classification and definitions by international consensus. Gut. 2013 Jan;62(1):102-11.

12. McMahon MJ, Pickford IR. Biochemical prediction of gallstones early in an attack of acute pancreatitis. Lancet. 1979 Sep 15;2(8142):541-3.

13. Anderson K, Brown LA, Daniel P, Connor SJ. Alanine transaminase rather than abdominal ultrasound alone is an important investigation to justify cholecystectomy in patients presenting with acute pancreatitis. HPB. 2010 Jun;12(5):342-7.

14. Güngör B, Cağlayan K, Polat C, Seren D, Erzurumlu $\mathrm{K}$, Malazgirt Z. The predictivity of serum biochemical markers in acute biliary pancreatitis. ISRN Gastroenterol. 2011;2011:279607. 